\title{
INSIGHTS
}

\section{Family reflections: pain in premature infants}

\author{
Nanon H.M. Labrie ${ }^{1}$ \\ Pediatric Research (2020) 87:176-177; https://doi.org/10.1038/s41390-019-0660-9
}

Seeing your child in pain is probably one of the most difficult things parents have to face. Usually, new parents get gently acquainted with the idea that their children will have to endure discomfort, pain, and disappointment from time to time-from a first fever temperature, to nighttime teething aches, and a first bruised knee (I think this one actually hurt me more than my daughter!). Yet, parents of premature infants do not receive such a gentle introduction into parenthood. They are confronted with their children's pain from their very first day at the Neonatal Intensive Care Unit (NICU).

My daughter was born when I was 26 weeks and 3 days pregnant. I developed a severe and early form of preeclampsia and my blood pressure was sky rocketing. To help prolong the pregnancy, I was treated with magnesium sulfate. Yet, by the time the hospital staff had managed to get my situation under control, my daughter started showing signs of serious distress. As her condition worsened rapidly, an emergency C-section was performed. I do not remember much, but how fearful I felt. Only few days prior, I had given a conference presentation (ironically, on the importance of information provision in doctor-patient communication) at a large, international congress, and now I had become a patient myself. More so, unexpectedly early, I had become a mother. Suffering from posttraumatic stress disorder, it took me days, weeks perhaps, to fully come to terms with my daughter's premature birth-as I felt my body had failed us both.

The first few days at the NICU my daughter thrived. But on her third night, she suffered from pulmonary hemorrhaging and had to be resuscitated. At 3 a.m., we were rushed from the maternity ward to the NICU, where all staff had gathered around her incubator. Alarm bells were ringing, lights were blinking. Our daughter was evidently in agony, and so were we. Thankfully, she survived, but in the weeks that followed, she had to receive multiple blood transfusions (so many, in fact, we lost count) and remained intubated to allow her to breathe steadily. Because of the tube, she could not produce sounds. The sight of her silent crying broke our hearts.

Our daughter stayed in the hospital for 103 days in total, 8 weeks of which at the NICU. Throughout this period, she received high levels of oxygen and breathing support. The continuous exposure to tubes and probes caused my daughter to become irritable when touched around her nose and mouth. Ultimately, she developed difficulties breastfeeding and bottle feeding. It took several months after we were released from the hospital before her feeding probe could be removed. The long nights at home, probe-feeding breast milk to our daughter, without the wise words of the NICU staff members to help and comfort us, were probably among the loneliest in our lives.

It has been 2 years since, and my daughter is a happy, healthy, and very chatty toddler, whose development continues to amaze us. Over time (and with plenty of patience), she slowly overcame the irritability to her nose and mouth and she now even enjoys mealtimes. Her lungs are still comparatively fragile, yet she is attending a regular kindergarten, climbing, and running, without any trouble.

Although it has been a long process, I have also recovered from preeclampsia. I am back at work-as a researcher of healthcare provider-patient communication. My professional expertise and personal experiences have led me to reflect on our NICU period from a communication perspective. As a researcher, I was familiar with studies which demonstrate that most patients have high information needs and that many patients, albeit fewer, also want to be involved in decision-making about treatment. As a patient and NICU parent, however, I quickly realized that, personally, I preferred to have as limited information as possible. That is, I surely desired sufficient information to engage in discussions about important treatment decisions and pain management. However, to cope with my emotions, I did not want to receive more information than strictly necessary. Thereby, I deviated from the "average" patient and parent. I did not always feel understood.

Research on information provision across medical settings often focuses on ensuring that patients and parents are sufficiently informed to cope with the situation at hand and to adequately make decisions about treatment. However, being sufficient informed is not a matter of information quantity. Depending on parents' personal information needs and preferences, adequate information provision may be a comparatively limited exchange of facts and knowledge about their child's health status. After all, while most parents may have high information needs, not all parents do. There is no "average" parent. Some parents may prefer verbal communication, other parents may prefer visual information-which they can reread in the quiet of their home.

In order for parents to recall crucial information, to empower them to engage in decision-making, and to avoid that they feel bystanders in the care of their own child, it is thus pivotal that healthcare providers tailor information to individual parents' needs. Doing so, timing may be everything: during the first few days at the NICU, parents may need more guidance and information than later on. To parents, the NICU is a complex and daunting environment to navigate-full of new and difficult jargon and procedures. For example, I was scared to change a diaper in the first few days, even with the help of an experienced nurse. My own condition, being both very ill and traumatized, made me less receptive to new information, despite that it concerned my beautiful, newborn daughter.

In my opinion, experts of neonatal care and communication researchers should join forces to (further) study what it implies to

${ }^{1}$ Athena Institute, Faculty of Science, Vrije Universiteit Amsterdam, Amsterdam, The Netherlands

Correspondence: Nanon H.M. Labrie (n.h.m.labrie@vu.nl)

Received: 29 October 2019 Accepted: 29 October 2019

Published online: 6 November 2019 
feel sufficiently informed for individual NICU parents, beyond presence as a desirable outcome. For, I personally believe preferred outcomes should be parental empowerment and parent-child attachment. Research should address the question what tailoring information precisely entails. Possibly, the simplest explanation would be to explicitly (and repeatedly) ask parents about their information needs, to document their preferences, and consistently respect these.

Being confronted with their child's pain and distress is hard for NICU parents, regardless of the extent to which they are informed about pain management. However, effective information provision is necessary when striving to empower parents to be actively present and involved in their child's care. I strongly believe that the key to adequate information provision is to adapt communication to parents' personal needs and preferences (in terms of information quantity, timing, means).
Providing tailored information will help encourage parents to voice their own preferences, opinions, and feelings as wellultimately leading to parents that are actively involved, present, and well attached to their child.

\section{FUNDING}

The author's work is funded through a personal research grant from the Dutch Research Council (NWO, VI.Veni.191S.032).

\section{ADDITIONAL INFORMATION}

Conflict of interest: The author declares no competing interests.

Publisher's note Springer Nature remains neutral with regard to jurisdictional claims in published maps and institutional affiliations. 